\title{
Residual implications derived from uninorms satisfying Modus Ponens
}

\author{
M. Mas, M. Monserrat, D. Ruiz-Aguilera, J. Torrens \\ Department of Mathematics and Computer Science \\ University of the Balearic Islands \\ Cra. de Valldemosa, $\mathrm{km} \mathrm{7,5}$ \\ 07122 Palma, Illes Balears, Spain \\ e-mails: \{mmg448, mma112, daniel.ruiz, jts224\}@uib.es
}

\begin{abstract}
Modus Ponens is a key property for fuzzy implication functions that are going to be used in fuzzy inference processes. In this paper it is investigated when fuzzy implication functions derived from uninorms via residuation, usually called $R U$ implications, satisfy the modus ponens with respect to a continuous t-norm $T$, or equivalently, when they are $T$-conditionals. For $R U$-implications it is proved that $T$-conditionality only depends on the underlying t-norm $T_{U}$ of the uninorm $U$ used to derive the residual implication and this fact leads to a lot of new solutions of the Modus Ponens property. Along the paper the particular cases when the uninorm lies in any of the most usual classes of uninorms are considered.
\end{abstract}

Keywords: Fuzzy implication function, residual implication, Modus Ponens, uninorm

\section{Introduction}

Fuzzy implication functions play a fundamental role in fuzzy logic and approximate reasoning. This kind of logical operations are essential in modelling all fuzzy conditionals and also in the inference process. Moreover, they are also useful in many application fields not only derived from the proper approximate reasoning, but also in other aspects as fuzzy subset-hood measures, fuzzy relational equations, fuzzy mathematical morphology, and computing with words among others. For this reason, investigations on fuzzy implication functions have been extensively developed in last decades even from the pure theoretical point of view, as it can be seen in the survey [18] and in the books [3, 4], entirely devoted to this kind of logical operations.

One of the main topics in this theoretical study consists on the investigation of additional properties of implication functions, properties that usually come from the concrete applications where implications functions are going to be applied. The study of each one of these additional properties usually leads to solve a functional equation (or inequality) involving fuzzy implication functions (see for instance Chapter 7 in [4] and the references therein).
One of these additional properties, that in this case comes from approximate reasoning, is known as the (generalized) Modus Ponens. In fact, forward inference schemes in approximate reasoning are usually based on the Modus Ponens that is carried out through the well known Compositional Rule of Inference (CRI) of Zadeh, based on the sup $-T$ composition, where $T$ is a t-norm (see for instance, Section 8.3 in [4]). Thus, if $I$ is a fuzzy implication function and $T$ is a t-norm, the Modus Ponens property for $I$ with respect to $T$ becomes the functional inequality:

$$
T(x, I(x, y)) \leq y \quad \text { for all } x, y \in[0,1]
$$

property that is also known as $T$-conditionality.

The Modus Ponens has been extensively studied in the literature by some authors (namely $[2,4,16$, 24, 25, 26, 27]). However, all these studies involve only the main classes of implication functions:

1. $R$-implications derived from (left-continuous) t-norms, $I_{T}(x, y)=$

$$
\sup \{z \in[0,1] \mid T(x, z) \leq y, x, y \in[0,1]\}
$$

2. $(S, N)$-implications derived from a t-conorm $S$ and a fuzzy negation $N$,

$$
I_{S, N}(x, y)=S(N(x), y), x, y \in[0,1]
$$

3. $Q L$-implications derived from a t-norm $T$, a tconorm $S$ and a fuzzy negation $N$,

$$
I_{S, N, T}(x, y)=S(N(x), T(x, y)), x, y \in[0,1] .
$$

On the other hand, note that there exist other kinds of implication functions like $D$-implications and Yager's implications. Moreover, some generalizations of $R,(S, N)$, and $Q L$-implications have been introduced, by substituting the t-norm and the tconorm by more general aggregation functions (for more details see [4] and also [19] with the references therein). One of these generalizations is based on uninorms obtaining the so-called $R U$-implications ([7]), $(U, N)$-implications ([5]), and even $Q L$ and $D$ implications derived from conjunctive and disjunctive uninorms $([15])$. 
For these kinds of implications the Modus Ponens has not been studied yet and this is the idea of the present paper. In particular we want to deal with the case of $R U$-implications, leaving the other cases for a future work. Specifically, we want to study the $T$-conditionality with respect to any continuous t-norm $T$ for the case of $R U$-implications. We will prove that there are a lot of them that satisfy the Modus Ponens with respect to any t-norm $T$, and we will characterize the special case when the t-norm $T$ and the underlying operations of the uninorm are continuous.

\section{Preliminaries}

We will suppose the reader to be familiar with the theory of t-norms, t-conorms and fuzzy negations (all necessary results and notations can be found in [11]). We also suppose that some basic facts on uninorms are known (see for instance [9]) as well as their most usual classes, that is, uninorms in $\mathcal{U}_{\text {min }}$ and $\mathcal{U}_{\max }([9])$, representable uninorms $([9])$, idempotent uninorms $([6,14,23])$ and uninorms continuous in the open unit square ([10]).

We recall here only some facts on implications and uninorms in order to stablish the necessary notation that we will use along the paper.

Definition 1 A binary operator $I:[0,1] \times[0,1] \rightarrow$ $[0,1]$ is said to be a fuzzy implication function, or an implication, if it satisfies:

(I1) $I(x, z) \geq I(y, z)$ when $x \leq y$, for all $z \in[0,1]$.

(I2) $I(x, y) \leq I(x, z)$ when $y \leq z$, for all $x \in[0,1]$.

(I3) $I(0,0)=I(1,1)=1$ and $I(1,0)=0$.

Note that, from the definition, it follows that $I(0, x)=1$ and $I(x, 1)=1$ for all $x \in[0,1]$ whereas the symmetrical values $I(x, 0)$ and $I(1, x)$ are not derived from the definition.

Definition $2 A$ uninorm is a two-place function $U:[0,1]^{2} \longrightarrow[0,1]$ which is associative, commutative, increasing in each place and such that there exists some element $e \in[0,1]$, called neutral element, such that $U(e, x)=x$ for all $x \in[0,1]$.

Evidently, a uninorm with neutral element $e=1$ is a t-norm and a uninorm with neutral element $e=0$ is a t-conorm. For any other value $e \in] 0,1[$ the operation works as a t-norm in the $[0, e]^{2}$ square, as a t-conorm in $[e, 1]^{2}$ and its values are between minimum and maximum in the set of points $A(e)$ given by

$$
A(e)=[0, e[\times] e, 1] \cup] e, 1] \times[0, e[.
$$

We will usually denote a uninorm with neutral element $e$ and underlying t-norm and t-conorm, $T$ and $S$, by $U \equiv\langle T, e, S\rangle$. For any uninorm it is satisfied that $U(0,1) \in\{0,1\}$ and a uninorm $U$ is called conjunctive if $U(1,0)=0$ and disjunctive when $U(1,0)=1$. On the other hand, let us recall the most studied classes of uninorms in the literature.

Theorem 1 ([9]) Let $U:[0,1]^{2} \rightarrow[0,1]$ be a uninorm with neutral element $e \in] 0,1[$.

(a) If $U(0,1)=0$, then the section $x \mapsto U(x, 1)$ is continuous except in $x=e$ if and only if $U$ is given by $U(x, y)=$

$$
\begin{cases}e T\left(\frac{x}{e}, \frac{y}{e}\right) & \text { if }(x, y) \in[0, e]^{2}, \\ e+(1-e) S\left(\frac{x-e}{1-e}, \frac{y-e}{1-e}\right) & \text { if }(x, y) \in[e, 1]^{2}, \\ \min (x, y) & \text { if }(x, y) \in A(e),\end{cases}
$$

where $T$ is a t-norm, and $S$ is a t-conorm.

(b) If $U(0,1)=1$, then the section $x \mapsto U(x, 0)$ is continuous except in $x=e$ if and only if $U$ is given by the same structure as above, changing minimum by maximum in $A(e)$.

The set of uninorms as in case (a) will be denoted by $\mathcal{U}_{\min }$ and the set of uninorms as in case (b) by $\mathcal{U}_{\max }$. We will denote a uninorm in $\mathcal{U}_{\min }$ with underlying t-norm $T$, underlying t-conorm $S$ and neutral element e as $U \equiv\langle T, e, S\rangle_{\min }$ and in a similar way, a uninorm in $\mathcal{U}_{\max }$ as $U \equiv\langle T, e, S\rangle_{\max }$.

Idempotent uninorms were characterized first in [6] for those with a lateral continuity and in [14] for the general case. An improvement of this last result was done in [23] as follows.

Theorem 2 ([23]) $U$ is an idempotent uninorm with neutral element $e \in[0,1]$ if and only if there exists a non increasing function $g:[0,1] \rightarrow[0,1]$, symmetric with respect to the identity function, with $g(e)=e$, such that $U(x, y)=$

$$
\left\{\begin{aligned}
\min (x, y) & \text { if } y<g(x) \text { or } \\
& \left(y=g(x) \text { and } x<g^{2}(x)\right), \\
\max (x, y) & \text { if } y>g(x) \text { or } \\
& \left(y=g(x) \text { and } x>g^{2}(x)\right), \\
x \text { or } y & \text { if } y=g(x) \text { and } x=g^{2}(x),
\end{aligned}\right.
$$

being commutative in the points $(x, y)$ such that $y=g(x)$ with $x=g^{2}(x)$.

Any idempotent uninorm $U$ with neutral element $e$ and associated function $g$, will be denoted by $U \equiv$ $\langle g, e\rangle_{\text {ide }}$ and the class of idempotent uninorms will be denoted by $\mathcal{U}_{\text {ide. }}$ Obviously, for any of these uninorms the underlying t-norm $T$ is the minimum and the underlying t-conorm $S$ is the maximum.

Definition 3 ([9]) Let e be in ]0,1[. A binary operation $U:[0,1]^{2} \rightarrow[0,1]$ is a representable uninorm if and only if there exists a strictly increasing function $h:[0,1] \rightarrow[-\infty,+\infty]$ with $h(0)=-\infty, h(e)=0$ and $h(1)=+\infty$ such that

$$
U(x, y)=h^{-1}(h(x)+h(y))
$$


for all $(x, y) \in[0,1]^{2} \backslash\{(0,1),(1,0)\}$ and $U(0,1)=$ $U(1,0) \in\{0,1\}$. The function $h$ is usually called an additive generator of $U$.

Remark 1 Recall that the are no continuous uninorms with neutral element $e \in] 0,1[$. In fact, representable uninorms were characterized as those uninorms that are continuous in $[0,1]^{2} \backslash\{(1,0),(0,1)\}$ (see [21]) as well as those that are strictly increasing in the open unit square (see [8]).

Any representable uninorm $U$ with neutral element $e$ and additive generator $h$, will be denoted by $U \equiv\langle h, e\rangle_{\text {rep }}$ and the class of representable uninorms will be denoted by $\mathcal{U}_{\text {rep }}$. For any of these uninorms the underlying t-norm $T$ is always strict and the underlying t-conorm $S$ is strict as well.

A more general class containing representable uninorms is given by those uninorms that are continuous in the open unit square $] 0,1\left[{ }^{2}\right.$. This class was characterized in [10] as follows.

Theorem 3 ([10] and [21] for the current version) Suppose $U$ is a uninorm continuous in $] 0,1\left[^{2}\right.$ with neutral element $e \in] 0,1[$. Then either one of the following cases is satisfied:

(a) There exist $u \in[0, e[, \lambda \in[0, u]$, two continuous t-norms $T_{1}$ and $T_{2}$ and a representable uninorm $R$ such that $U$ can be represented as $U(x, y)=$

$$
\begin{cases}\lambda T_{1}\left(\frac{x}{\lambda}, \frac{y}{\lambda}\right) & \text { if } x, y \in[0, \lambda], \\ \lambda+(u-\lambda) T_{2}\left(\frac{x-\lambda}{u-\lambda}, \frac{y-\lambda}{u-\lambda}\right) & \text { if } x, y \in[\lambda, u], \\ u+(1-u) R\left(\frac{x-u}{1-u}, \frac{y-u}{1-u}\right) & \text { if } x, y \in] u, 1[, \\ 1 & \text { if } \min (x, y) \in] \lambda, 1] \\ & \text { and } \max (x, y)=1, \\ \lambda \text { or } 1 & \text { if }(x, y)=(\lambda, 1) \\ & \text { or }(x, y)=(1, \lambda), \\ \min (x, y) & \text { elsewhere. }\end{cases}
$$

(b) There exist $v \in] e, 1], \omega \in[v, 1]$, two continuous t-conorms $S_{1}$ and $S_{2}$ and a representable uninorm $R$ such that $U$ can be represented as $U(x, y)=$

$$
\begin{cases}v+(\omega-v) S_{1}\left(\frac{x-v}{\omega-v}, \frac{y-v}{\omega-v}\right) & \text { if } x, y \in[v, \omega], \\ \omega+(1-\omega) S_{2}\left(\frac{x-\omega}{1-\omega}, \frac{y-\omega}{1-\omega}\right) & \text { if } x, y \in[\omega, 1], \\ v R\left(\frac{x}{v}, \frac{y}{v}\right) & x, y \in] 0, v[, \\ 0 & \text { if } \max (x, y) \in[0, \omega[ \\ & \text { and } \min (x, y)=0, \\ \omega \text { or } 0 & \text { if }(x, y)=(0, \omega) \\ & \text { or }(x, y)=(\omega, 0), \\ \max (x, y) & \text { elsewhere. }\end{cases}
$$

The class of all uninorms continuous in $] 0,1\left[^{2}\right.$ will be denoted by $\mathcal{U}_{\text {cos }}$. A uninorm as in (1) will be denoted by $U \equiv\left\langle T_{1}, \lambda, T_{2}, u,(R, e)\right\rangle_{\mathrm{cos}, \min }$ and the class of all uninorms continuous in the open unit square of this form will be denoted by $\mathcal{U}_{\text {cos,min }}$. Analogously, a uninorm as in (2) will be denoted by $U \equiv\left\langle(R, e), v, S_{1}, \omega, S_{2}\right\rangle_{\cos , \max }$ and the class of all uninorms continuous in the open unit square of this form will be denoted by $\mathcal{U}_{\mathrm{cos}, \max }$. For any uninorm $U \equiv\left\langle T_{1}, \lambda, T_{2}, u,(R, e)\right\rangle_{\mathrm{cos}, \min }$, the underlying t-norm of $U$ is given by an ordinal sum of three t-norms, $T_{1}, T_{2}$ and a strict t-norm, whereas the underlying t-conorm is always strict. Similarly, for any uninorm $U \equiv\left\langle(R, e), v, S_{1}, \omega, S_{2}\right\rangle_{\cos , \max }$, the underlying t-norm of $T$ is always strict, whereas the underlying t-conorm is given by an ordinal sum of three t-conorms, a strict t-conorm, $S_{1}$, and $S_{2}$.

On the other hand, different classes of implications derived from uninorms have been studied. We recall here $R U$-implications.

Definition 4 Let $U$ be a uninorm. The residual operation derived from $U$ is the binary operation given by $I_{U}(x, y)=$

$$
\sup \{z \in[0,1] \mid U(x, z) \leq y\} \text { for all } x, y \in[0,1] .
$$

Proposition 4 ([r]) Let $U$ be a uninorm and $I_{U}$ its residual operation. Then $I_{U}$ is an implication if and only if the following condition holds

$$
U(x, 0)=0 \text { for all } x<1 .
$$

In this case $I_{U}$ is called an $R U$-implication.

This includes all conjunctive uninorms but also many disjunctive ones, for instance in the classes of representable uninorms (see [7]), idempotent uninorms (see [20]), and uninorms continuous in the unit open square (see [22]). However, when we deal with left-continuous uninorms $U$ we clearly have that $U$ satisfies condition (3) if and only if it is conjunctive.

Some properties of $R U$-implications have been studied involving the main classes of uninorms, those previously stated: uninorms in $\mathcal{U}_{\text {min }}$, representable uninorms, idempotent uninorms and uninorms continuous in the open unit square (for more details see $[1,4,7,17,20,22])$. However, although the strong interest of the Modus Ponens property, its study is not among the properties investigated for implications derived from uninorms. Let us recall the definition of the Modus Ponens in the framework of fuzzy logic.

Definition 5 Let I be an implication function and $T$ a t-norm. It is said that I satisfies the Modus Ponens property with respect to $T$, or that $I$ is a $T$-conditional if

$$
T(x, I(x, y)) \leq y \quad \text { for all } x, y \in[0,1] .
$$

A well known general result on $T$-conditionality was proved in [24].

Proposition 5 Let $I$ be an implication function and $T$ a left-continuous t-norm. Then $I$ is a $T$ conditional if and only if $I \leq I_{T}$, where $I_{T}$ denotes the residual implication derived from $T$. 


\section{3. $R U$-implications that are $T$-conditionals}

In this section we want to deal with the case of $R U$ implications. Thus, the main goal of this section is to characterize when an $R U$-implication derived from a uninorm $U$ is a $T$-conditional for a t-norm $T$, specially when $T$ is continuous. All along this section it will be understood that any considered uninorm $U$ satisfies $U(x, 0)=0$ for all $x<1$, in order to ensure that the corresponding residual $I_{U}$ is an $R U$-implication, according to Proposition 4.

Proposition 6 Let $U$ be a uninorm with neutral element $e \in] 0,1\left[\right.$ and underlying $t$-norm $T_{U}$, and let $I_{U}$ be the corresponding $R U$-implication. Then the following items are equivalent:

i) $I_{U}$ is a T-conditional.

ii) $I_{U}$ satisfies Equation (4) for all $y<x<e$.

iii) The inequality

$$
T\left(x, e I_{T_{U}}\left(\frac{x}{e}, \frac{y}{e}\right)\right) \leq y
$$

holds for all $x, y$ such that $y<x<e$, where $I_{T_{U}}$ denotes the residual implication derived from the $t$-norm $T_{U}$.

This result proves that the underlying t-conorm $S_{U}$ of the uninorm $U$ and the values of $U$ in the region $A(e)$ are not relevant in order $I_{U}$ to be a $T$-conditional. Only the underlying t-norm $T_{U}$ is relevant and the inequality corresponding to $T$ conditionality only needs to be checked in the region

$$
R_{e}=\left\{(x, y) \in[0,1]^{2} \mid y<x<e\right\} .
$$

The region $R_{e}$ is pictured in Figure 3 .

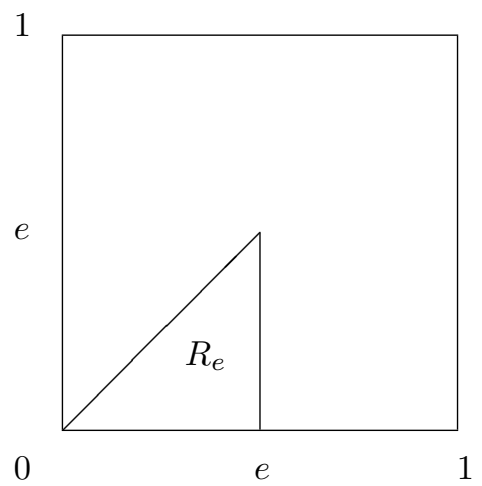

Figure 1: Region $R_{e}$.

Thus, we continue our study depending on how the underlying t-norm $T_{U}$ is. Note that if $T(e, e)=$ 0 , then condition iii) in Proposition 6 is always satisfied. Then, any t-norm $T_{U}$ (continuous or not) will work in this case. From now on, we will restrict ourselves to the case when $T_{U}$ is continuous. Taking into account the classification of continuous t-norms (see for instance [11]), we will divide our study in three steps respectively devoted to the cases when $T_{U}=\min , T_{U}$ is Archimedean or $T_{U}$ is given by an ordinal sum.

Let us begin with the case when $T_{U}=\min$.

Proposition 7 Let $U$ be a uninorm with neutral element $e \in] 0,1\left[\right.$ and underlying t-norm $T_{U}$ given by the minimum. Then the $R U$-implication $I_{U}$ is a $T$-conditional for any $t$-norm $T$. In particular, this $i s$ the case for any idempotent uninorm $U$.

Example 1 Let $N$ be a strong negation. Among the class of idempotent uninorms, an important example is given by uninorms whenever $g=N$ (see [20]), that is, they have the form

$$
U(x, y)= \begin{cases}\min (x, y) & \text { if } y<N(x) \\ \max (x, y) & \text { if } y>N(x) \\ \min (x, y) \text { or } \max (x, y) & \text { otherwise }\end{cases}
$$

being commutative in the points $(x, y)$ such that $y=N(x)$. In these cases the corresponding $R U$ implication is given by

$$
I_{U}(x, y)= \begin{cases}\min (N(x), y) & \text { if } y<x \\ \max (N(x), y) & \text { if } y \geq x\end{cases}
$$

From the proposition above, all these implications are $T$-conditionals for any t-norm $T$. Figure 3 shows the idempotent uninorm $U \equiv\left\langle N, \frac{1}{2}\right\rangle_{i d e}$ and its $R U$-implication given in this example when the considered negation is the classical one $N_{c}(x)=$ $1-x$.

Let us now deal with the case when the underlying t-norm $T_{U}$ is Archimedean and the t-norm $T$ is continuous. It is well known that when a t-norm is Archimedean it is represented by a decreasing additive generator $\varphi:[0,1] \rightarrow[0,+\infty]$, with $\varphi(0)=+\infty$ if the t-norm is strict, and with $\varphi(0)=1$ when the t-norm is nilpotent. Moreover, in this last case the function $N(x)=\varphi^{-1}(1-\varphi(x))$ for all $x \in[0,1]$ is a strong negation usually called the associated negation of the nilpotent t-norm $T$. Let us consider both cases separately.

Proposition 8 Let $U$ be a uninorm with neutral element $e \in] 0,1\left[\right.$ and underlying t-norm $T_{U}$ strict. Let $I_{U}$ be the $R U$-implication derived from $U$ and $T$ a continuous t-norm.

i) If $I_{U}$ is a T-conditional, then there exists $a \geq e$ such that $T$ is an ordinal sum of the form $T=$ $\left(\left\langle 0, a, T_{a}\right\rangle,\left\langle a, 1, T_{1}\right\rangle\right)$, where $T_{a}$ is Archimedean and $T_{1}$ continuous.

ii) Let $\varphi$ and $\varphi_{a}$ be the additive generators of $T_{U}$ and $T_{a}$, respectively. Then $I_{U}$ is a $T$ conditional if and only if the function $g$ defined from $[0,+\infty]$ to $\left[\varphi_{a}\left(\frac{e}{a}\right), \varphi_{a}(0)\right]$ given by the expression $g(u)=\varphi_{a}\left(\frac{e}{a} \varphi^{-1}(u)\right)$ is sub-additive. 

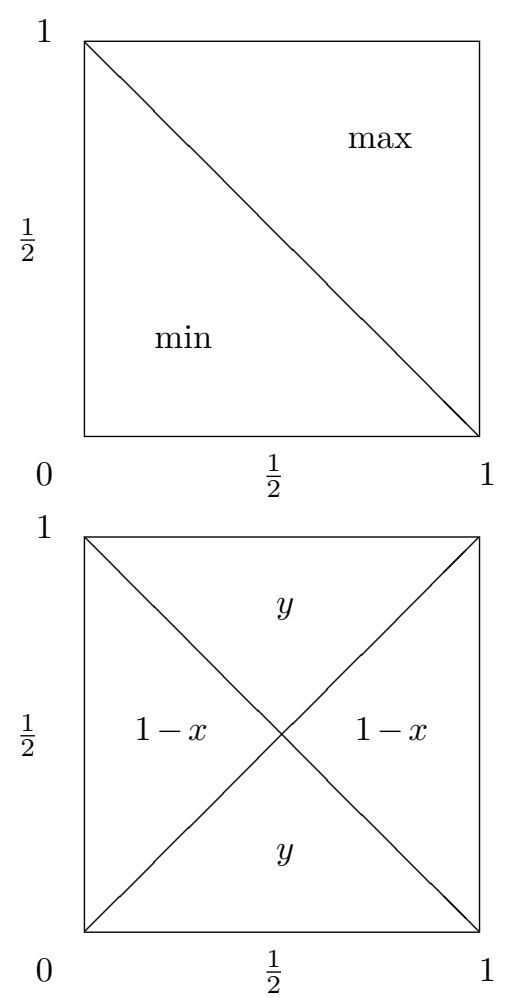

Figure 2: Structure of $U \equiv\left\langle N, \frac{1}{2}\right\rangle_{\text {ide }}$ (top) and $I_{U}$ (bottom) with $N_{c}(x)=1-x$.

Remark 2 i) Note that when $a=e$, the result above can be related to the results about $T$ conditionality for residual implications derived from continuous t-norms given in [24]. Specifically, we can derive that $I_{U}$ is a T-conditional if and only $T=\left(\left\langle 0, e, T_{e}\right\rangle,\left\langle e, 1, T_{1}\right\rangle\right)$, where $T_{e}$ is Archimedean, $T_{1}$ continuous and $I_{T_{U}}$ is a $T_{e}$-conditional.

ii) Of course that one can take $a=1$ in the proposition above obtaining $R U$-implications that are $T$-conditional for Archimedean t-norms $T$.

iii) Note that uninorms with $T_{U}$ strict include, but are not limited to, all representable uninorms as well as those uninorms continuous in $] 0,1\left[^{2}\right.$ lying in $\mathcal{U}_{\mathrm{cos}, \max }$. In addition, the subset of all uninorms with $T_{U}$ and $S_{U}$ strict have been recently characterized in [13].

Example 2 Let us take, for instance, the conjunctive uninorm given by $U(x, y)=$

$$
\begin{cases}0 & \text { if }(x, y) \in\{(1,0),(0,1)\}, \\ \frac{x y}{x y+(1-x)(1-y)} & \text { otherwise, }\end{cases}
$$

whose residual implication $I_{U}$ is given by

$$
I_{U}(x, y)= \begin{cases}1 & \text { if }(x, y) \in\{(0,0),(1,1)\} \\ \frac{(1-x) y}{x+y-2 x y} & \text { otherwise }\end{cases}
$$

and take also $T=T_{\mathbf{P}}$ the product $t$-norm. It is easy to see that in this case $I_{U}$ is a T-conditional.
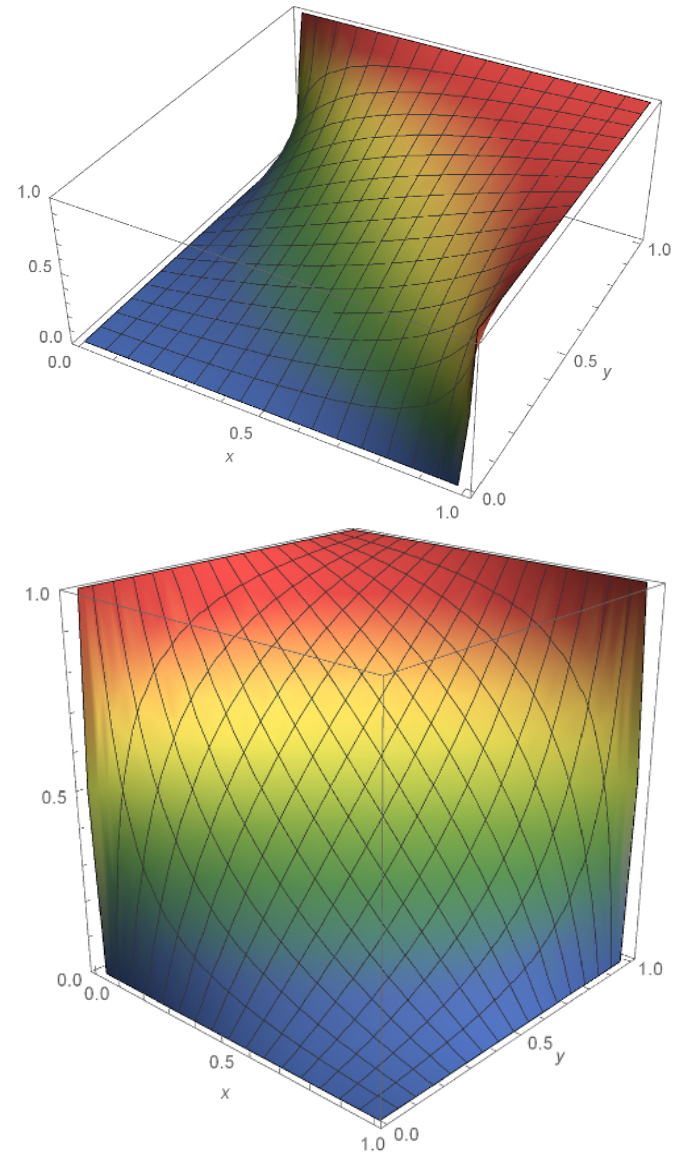

Figure 3: Representable uninorm $U$ with additive generator $h(x)=\log \left(\frac{x}{1-x}\right)$ (top) and its residual implication $I_{U}$ (bottom).

Namely, it is well known that $U$ is a representable uninorm with neutral element $e=\frac{1}{2}$ and additive generator $h(x)=\log \left(\frac{x}{1-x}\right)$. In this case, the underlying t-norm $T_{U}$ is strict with additive generator given by $\varphi(x)=\log \left(\frac{2-x}{x}\right)$ (see [9] or Section 10.2 in [11]). Moreover, this situation corresponds to take $a=1$ and $\varphi_{1}(x)=-\log (x)$ in Proposition 8 and consequently the corresponding function $g$ is given by

$$
\begin{aligned}
g(x) & =\varphi_{1}\left(\frac{1}{2} \varphi^{-1}(x)\right)=-\log \left(\frac{1}{2} \frac{2}{1+e^{x}}\right) \\
& =\log \left(1+e^{x}\right),
\end{aligned}
$$

which is clearly sub-additive. In Figure 3 uninorm $U$ and its residual implication $I_{U}$ have been depicted.

Proposition 9 Let $U$ be a uninorm with neutral element $e \in] 0,1\left[\right.$ and underlying t-norm $T_{U}$ nilpotent with associated negation $N_{U}$. Let $I_{U}$ be the $R U$-implication derived from $U$ and $T$ a continuous t-norm.

i) If $I_{U}$ is a T-conditional with respect to $T$, then there exists $a \geq e$ such that $T$ is an ordinal sum 
of the form $T=\left(\left\langle 0, a, T_{a}\right\rangle,\left\langle a, 1, T_{1}\right\rangle\right)$, where $T_{a}$ is nilpotent with associated negation $N_{a}$ such that $N_{a}$ covers $N_{U}$, i.e., $e N_{U}\left(\frac{x}{e}\right) \leq a N_{a}\left(\frac{x}{a}\right)$ for all $x \in[0, e]$, and $T_{1}$ is continuous.

ii) Let $\varphi$ and $\varphi_{a}$ be the additive generators of $T_{U}$ and $T_{a}$, respectively. Then $I_{U}$ is a $T$ conditional with respect to $T$ if and only if the function $g:[0,1] \rightarrow\left[\varphi_{a}\left(\frac{e}{a}\right), 1\right]$ given by $g(u)=$ $\varphi_{a}\left(\frac{e}{a} \varphi^{-1}(u)\right)$ is sub-additive.

Remark 3 i) Note that in the previous proposition, the fact that $g$ is subadditive already ensures that the corresponding negations $N_{a}$ and $N_{U}$ are such that $N_{a}$ covers $N_{U}$. Namely, we have that $N_{a}$ covers $N_{U}$ if and only if

$$
e \varphi^{-1}\left(1-\varphi\left(\frac{x}{e}\right)\right) \leq a \varphi_{a}^{-1}\left(1-\varphi_{a}\left(\frac{x}{a}\right)\right)
$$

but taking the change $x=e \varphi^{-1}(z)$, this is equivalent to

$$
g(1-z) \geq 1-g(z)
$$

which is clearly satisfied when $g$ is sub-additive.

ii) Again note that when $a=e$, the result above can be stated as follows: $I_{U}$ is a T-conditional if and only $T=\left(\left\langle 0, e, T_{e}\right\rangle,\left\langle e, 1, T_{1}\right\rangle\right)$, where $T_{e}$ is Archimedean, $T_{1}$ continuous and $I_{T_{U}}$ is a $T_{e}$-conditional. Of course $a=1$ can be taken in the proposition above obtaining $R U$-implications that are T-conditional for nilpotent $t$ norms $T$.

iii) Recall that uninorms $U$ with $T_{U}$ nilpotent and $S_{U}$ Archimedean were characterized in [12] and [13].

Example 3 Let $U$ be a uninorm in $\mathcal{U}_{\text {min }}$ with neutral element $e=\frac{1}{2}$ and underlying t-norm $T_{U}=T_{\mathbf{L}}$ the Eukasiewicz t-norm, that is, $U$ is given by the expression

$$
U(x, y)= \begin{cases}\max \left(0, x+y-\frac{1}{2}\right) & \text { if }(x, y) \in\left[0, \frac{1}{2}\right]^{2}, \\ \frac{1+S_{U}(2 x-1,2 y-1)}{2} & \text { if }(x, y) \in\left[\frac{1}{2}, 1\right]^{2}, \\ \min (x, y) & \text { otherwise, }\end{cases}
$$

where $S_{U}$ can be any t-conorm. In this case $I_{U}$ is given by (see [7]):

$$
I_{U}(x, y)= \begin{cases}1 & \text { if } x<\frac{1}{2} \text { and } x \leq y, \\ \frac{1}{2}-x+y & \text { if } x<\frac{1}{2} \text { and } x>y, \\ y & \text { if } y \leq \frac{1}{2} \leq x, \\ \frac{1}{2} & \text { if } \frac{1}{2} \leq y<x, \\ \frac{1+R_{S_{U}}(2 x-1,2 y-1)}{2} & \text { if } \frac{1}{2} \leq x \leq y,\end{cases}
$$

where $R_{S_{U}}(x, y)=\sup \left\{z \in[0,1] \mid S_{U}(x, z) \leq y\right\}$. Then $I_{U}$ is always a $T_{\mathbf{L}}$-conditional because, using Proposition 9, we have $a=1$ and $\varphi(x)=\varphi_{1}(x)=$ $1-x$, obtaining $g(x)=\frac{x+1}{2}$ which is clearly subadditive. In Figure 4 we can see the structure of this general uninorm in $\mathcal{U}_{\min }$ as in this example and the corresponding $R U$-implication.
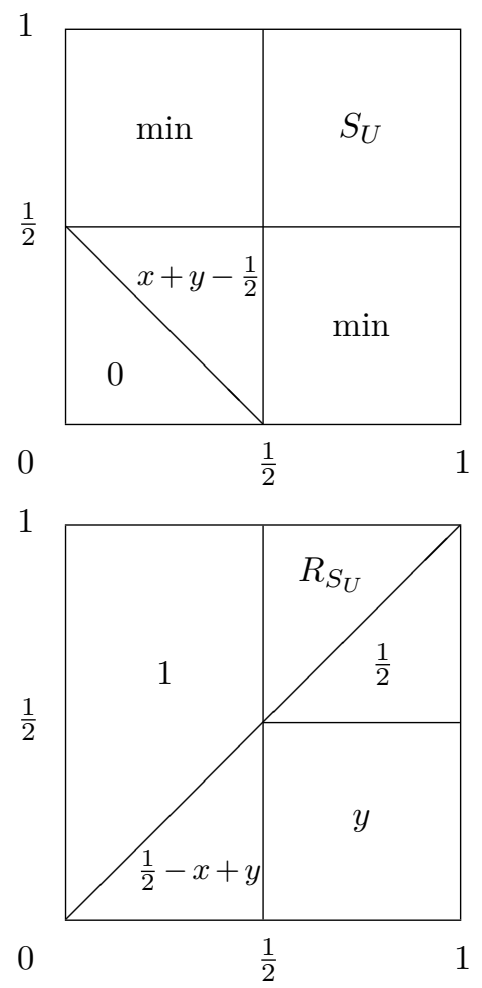

Figure 4: Structure of $U$ (top) and $I_{U}$ (bottom) when $U \in \mathcal{U}_{\min }, T_{U}=T_{\mathbf{L}}$ and $e=\frac{1}{2}$.

Proposition 10 Let $U$ be a uninorm with neutral element $e \in] 0,1[$ and underlying $t$-norm given by the ordinal sum $T_{U}=\left(\left\langle\frac{a_{i}}{e}, \frac{b_{i}}{e}, T_{i}\right\rangle\right)_{i \in I}$ with $0 \leq a_{i}<b_{i} \leq$ $e$ and $T_{i}$ Archimedean for all $i \in I$. Let $I_{U}$ be the $R U$-implication derived from $U$ and $T$ a continuous t-norm. Then $I_{U}$ is a T-conditional if and only if the following items hold:

i) The set of idempotent elements of $T$, that are less than or equal to $e$, are contained in $[0, e] \backslash$ $\left\{\cup_{i \in I}\left(a_{i}, b_{i}\right)\right\}$.

ii) The t-norm $T$ is an ordinal sum of the form $T=\left(\left\langle c_{j}, d_{i}, T_{j}^{\prime}\right\rangle\right)_{j \in J}$ in such a way that for all $i \in I$ there exists a $j \in J$ such that

$-\left(a_{i}, b_{i}\right) \subseteq\left(c_{j}, d_{j}\right)$, and if $T_{i}$ is nilpotent with associated negation $N_{i}$ for some $i \in I$ and $a_{i}=c_{j}$ then $T_{j}^{\prime}$ must be also nilpotent with associated negation $N_{j}$ such that $N_{j}$ covers $N_{i}$.

- If $\varphi_{i}$ and $\varphi_{j}$ are the respective additive generators of $T_{i}$ and $T_{j}^{\prime}$, then the function $g_{i j}$ that is defined from $\left[0, \varphi_{i}(0)\right]$ to $\left[\varphi_{j}\left(\frac{b_{i}-c_{j}}{d_{j}-c_{j}}\right), \varphi_{j}\left(\frac{a_{i}-c_{j}}{d_{j}-c_{j}}\right)\right]$ given by

$$
g_{i j}(u)=\varphi_{j}\left(\frac{a_{i}+\left(b_{i}-a_{i}\right) \varphi_{i}^{-1}(u)-c_{j}}{d_{j}-c_{j}}\right)
$$

is sub-additive.

From the previous result we can easily derive the following result. 
Proposition 11 Let $U$ be a uninorm with neutral element $e \in] 0,1[$ and underlying $t$-norm given by the ordinal sum $T_{U}=\left(\left\langle\frac{a_{i}}{e}, \frac{b_{i}}{e}, T_{i}\right\rangle\right)_{i \in I}$ with $0 \leq a_{i}<b_{i} \leq$ $e$ and $T_{i}$ Archimedean for all $i \in I$. Let $T$ be the ordinal sum $T=\left(\left\langle a_{i}, b_{i}, T_{i}^{\prime}\right\rangle\right)_{i \in I}$ with $T_{j}^{\prime}$ Archimedean for all $i \in I$ and suppose that $I_{T_{i}^{\prime}}$ is $T_{i}$-conditional for all $i \in I$. Then $I_{U}$ is a T-conditional.

Example 4 Let $U$ be a uninorm in $\mathcal{U}_{\text {min }}$ with neutral element $e=\frac{1}{2}$ and underlying $t$-norm $T_{U}$ given by the ordinal sum $T_{U}=\left(\left\langle 0, \frac{1}{2}, T_{\mathbf{P}}\right\rangle,\left\langle\frac{1}{2}, 1, T_{\mathbf{L}}\right\rangle\right)$ that $i s, U$ is given by the expression

$$
U(x, y)= \begin{cases}4 x y & \text { if }(x, y) \in\left[0, \frac{1}{4}\right]^{2}, \\ \max \left(\frac{1}{4}, x+y-\frac{1}{2}\right) & \text { if }(x, y) \in\left[\frac{1}{4}, \frac{1}{2}\right]^{2}, \\ \frac{1+S_{U}(2 x-1,2 y-1)}{2} & \text { if }(x, y) \in\left[\frac{1}{2}, 1\right]^{2}, \\ \min (x, y) & \text { otherwise, }\end{cases}
$$

where $S_{U}$ can be any t-conorm.

Let us consider the t-norm $T$ given by the expression

$$
T(x, y)= \begin{cases}T_{\mathbf{P}} & \text { if }(x, y) \in\left[0, \frac{1}{2}\right]^{2}, \\ T_{\mathbf{L}} & \text { if }(x, y) \in\left[\frac{1}{2}, 1\right]^{2}, \\ \min (x, y) & \text { otherwise. }\end{cases}
$$

Using Proposition 11, $I_{U}$ is a T-conditional because, $I_{T_{\mathbf{P}}}$ is a $T_{\mathbf{P}}$-conditional and $I_{T_{\mathbf{L}}}$ is a $T_{\mathbf{L}^{-}}$ conditional.

In Figure 5 we can see the structure of this general uninorm in $\mathcal{U}_{\min }$ as in this example and the corresponding $R U$-implication.

\section{Conclusions and future work}

Forward inference schemes in approximate reasoning are based on the Modus Ponens property, also called T-conditionality. Thus, fuzzy implication functions used in the inference process of any fuzzy rule based system are required to satisfy this property, which becomes essential in approximate reasoning and fuzzy control. Fixed a continuous tnorm $T$ modelling the conjunction, we studied in this paper which fuzzy implication functions satisfy $T$-conditionality among a special kind of implications derived from uninorms: $R U$-implications. In this case we have characterized all the solutions of the Modus Ponens property with respect to a continuous t-norm $T$ and from these characterizations we obtain a lot of new fuzzy implication functions satisfying the $T$-conditionality.

Moreover, we want to extend this study to the cases of other classes of fuzzy implication functions derived from uninorms, like $(U, N)$-implications and $Q L$ and $D$-implications. It is worth to point out that we have already started with the case of $(U, N)$ implications obtaining again a lot of new solutions. Moreover, contrariously to what happens with $R U$-implications, $T$-conditionality for $(U, N)$ implications only depends on the underlying tconorm $S_{U}$ and only in some cases, depending on
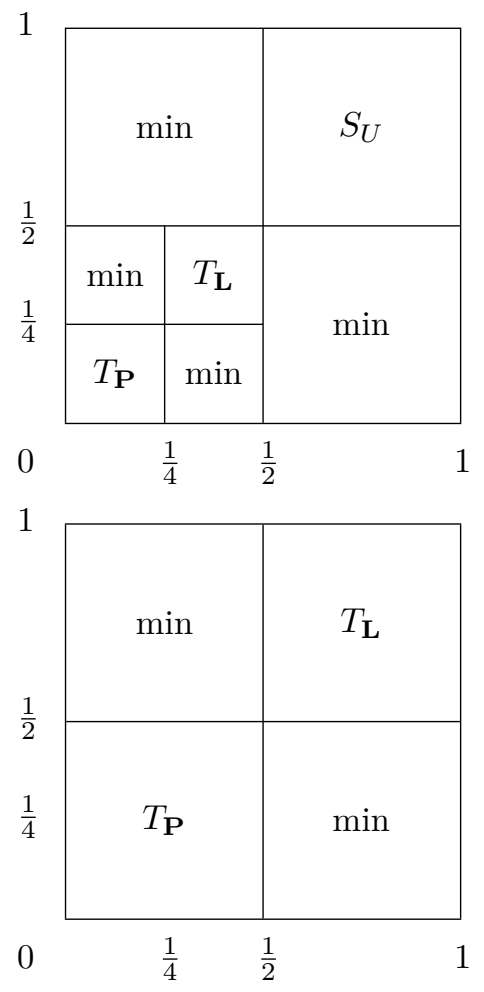

Figure 5: Structure of $U$ (top) and $T$ (bottom) such that $U \in \mathcal{U}_{\min }$, and $I_{U}$ is a $T$-conditional.

the value

$$
\alpha_{N}=\inf \{z \in[0,1] \mid N(z)=e\},
$$

where $N$ is the negation used to derive the corresponding $(U, N)$-implication, and $e$ is the neutral element of the uninorm $U$. Another possible extension that deserves to be investigated is related to the Modus Ponens with respect to a conjunctive uninorm $U$ instead of a continuous t-norm $\mathrm{T}$.

Of course that, as future work, it should be also included the study of the Modus Tollens for all these classes of implications.

\section{Acknowledgments}

This paper has been partially supported by the Spanish grant TIN2013-42795-P.

\section{References}

[1] I. Aguiló, J. Suñer, J. Torrens, A characterization of residual implications derived from leftcontinuous uninorms, Information Sciences, Vol. 180(20) (2010) 3992-4005.

[2] C. Alsina, E. Trillas, When $(S, N)$-implications are $\left(T, T_{1}\right)$-conditional functions?, Fuzzy Sets and Systems, Vol. 134, 305-310 (2003).

[3] M. Baczyński, G. Beliakov, H. Bustince-Sola, A. Pradera (Eds.), Advances in Fuzzy Implication Functions, Studies in Fuzziness and Soft 
Computing, vol. 300, Springer, Berlin Heidelberg, 2013.

[4] M. Baczyński, B. Jayaram, Fuzzy Implications, Studies in Fuzziness and Soft Computing, vol. 231, Springer, Berlin Heidelberg, 2008.

[5] M. Baczyński, B. Jayaram, (U,N)-implications and their characterizations, Fuzzy Sets and Systems, Vol. 160, 2049-2062 (2009).

[6] B. De Baets, Idempotent uninorms, European J. Oper. Res., Vol. 118 (1999) 631-642.

[7] B. De Baets, J. C. Fodor, Residual operators of uninorms, Soft Computing, Vol. 3, 89-100 (1999).

[8] J. Fodor, B. De Baets, A single-point characterization of representable uninorms, Fuzzy Sets and Systems, Vol. 202, 89-99 (2012).

[9] J. C. Fodor, R. R. Yager and A. Rybalov, Structure of Uninorms, Int. J. of Uncertainty, Fuzziness and Knowledge-based Systems, Vol. 5 (1997) 411-427.

[10] S. Hu and Z. Li, The structure of continuous uninorms. Fuzzy Sets and Systems, Vol. 124 (2001) 43-52.

[11] E.P. Klement, R. Mesiar and E. Pap, Triangular norms, Kluwer Academic Publishers, Dordrecht, 2000.

[12] G. Li, H.W. Liu, J. Fodor, Single-point characterization of uninorms with nilpotent underlying t-norm and t-conorm, Int. J. of Uncertainty, Fuzziness and Knowledge-based Systems, Vol. 22 (2014) 591-604.

[13] G. Li, H.W. Liu, Distributivity and conditional distributivity of a uninorm with continuous underlying operators over a continuous t-conorm, accepted in Fuzzy Sets and Systems.

[14] J. Martín, G. Mayor, J. Torrens, On locally internal monotonic operators, Fuzzy Sets and Systems, Vol. 137 (2003) 27-42.

[15] M. Mas, M. Monserrat, J. Torrens, Two types of implications derived from uninorms, Fuzzy Sets and Systems, Vol. 158, 2612-2626 (2007).

[16] M. Mas, M. Monserrat, J. Torrens, Modus Ponens and Modus Tollens in discrete implications, International Journal of Approximate Reasoning, Vol. 49, 422-435 (2008).

[17] M. Mas, M. Monserrat, J. Torrens, A characterization of $(U, N), R U, Q L$ and $D$-implications derived from uninorms satisfying the law of importation, Fuzzy Sets and Systems, Vol. 161 (2010) 1369-1387.

[18] M. Mas, M. Monserrat, J. Torrens, E. Trillas, A survey on fuzzy implication functions, IEEE Transactions on Fuzzy Systems, Vol. 15(6), 1107-1121 (2007).

[19] S. Massanet, J. Torrens, An overview of construction methods of fuzzy implications, in [3], pp. 1-30 (2013).

[20] D. Ruiz and J. Torrens, Residual implications and co-implications from idempotent uninorms, Kybernetika, Vol. 40 (2004) 21-38.
[21] D. Ruiz, J. Torrens, Distributivity and conditional distributivity of a uninorm and a continuous t-conorm. IEEE Transactions on Fuzzy Systems 14 (2006) 180-190.

[22] D. Ruiz-Aguilera and J. Torrens, $S-$ and $R$ - implications from uninorms continuous in ]0,1[2 and their distributivity over uninorms, Fuzzy Sets and Systems, Vol. 160 (2009) 832852.

[23] D. Ruiz-Aguilera, J. Torrens, B. De Baets, J. Fodor, Some remarks on the characterization of idempotent uninorms, in: E. Hüllermeier, R. Kruse, F. Hoffmann (Eds.), Computational Intelligence for Knowledge-Based Systems Design, Lecture Notes in Computer Science, vol. 6178, Springer, Berlin, Heidelberg, 2010, pp. 425-434.

[24] E. Trillas, C. Alsina, A. Pradera, On MPTimplication functions for fuzzy logic, Revista de la Real Academia de Ciencias. Serie A. Matemáticas (RACSAM), Vol 98(1), 259-271 (2004).

[25] E. Trillas, C. Alsina, E. Renedo, A. Pradera, On contra-symmetry and MPT-conditionality in fuzzy logic, International Journal of Intelligent Systems, Vol 20, 313-326 (2005).

[26] E. Trillas, C. Campo, S. Cubillo, When QMoperators are implication functions and conditional fuzzy relations, International Journal of Intelligent Systems, Vol 15, 647-655 (2000).

[27] E. Trillas, L. Valverde, On Modus Ponens in fuzzy logic, in Proceedings of the 15th International Symposium on Multiple-Valued Logic, Kingston, Canada, pp. 294-301 (1985). 